\title{
Los ácidos grasos omega-3 en la prevención de la enfermedad de Alzheimer
}

\author{
Javier Caballer García \\ Psiquiatra. CSM III Oviedo. Servicios de Salud Mental del Principado De Asturias
}

\section{Resumen}

Introducción y objetivos: los ácidos omega-3 son grasas poliinsaturadas que deben aportarse a través de la dieta, encontrándose de manera preformada fundamentalmente en las variedades del pescado azul. Los ácidos omega-3 se hallan en las membranas lipídicas neuronales en gran proporción, aportando estabilidad a las mismas y optimizando la comunicación sináptica. Una dieta pobre en ácidos omega-3, que se está convirtiendo una tendencia en los hábitos alimentarios occidentales en los últimos tiempos, podría figurar como un factor de riesgo para la aparición de alteraciones cognitivas. Este artículo de revisión trata de valorar la influencia de los ácidos omega-3 en la demencia (abordándose también el declive cognitivo y la situación de deterioro cognitivo ligero).

Desarrollo: se presenta una selección de datos de carácter neurobiológico, epidemiológico y clínico, a través de la revisión de las publicaciones más importantes en este sentido.

Conclusiones: la mayoría de los estudios de corte epidemiológico y algunos ensayos clínicos encuentran que una dieta rica en ácidos omega-3 se podría correlacionar con un enlentecimiento en el declive cognitivo normal asociado a la edad e incluso en pacientes ancianos diagnosticados de deterioro cognitivo ligero, lo que puede influir además en la disminución de la incidencia del diagnóstico de demencia. Sin embargo, los datos existentes en la actualidad no permiten encontrar una utilidad de los mismos en demencias ya establecidas.

El consumo frecuente de pescado azul es muy recomendable, y se debe considerar su aportación a modo de suplemento en el caso de que esto no ocurra.

(Alzheimer. Real Invest Demenc. 2011;49:12-18)

Palabras clave: ácidos omega-3, demencia, depresión, enfermedad de Alzheimer, grasas poliinsaturadas, psicogeriatría.

\section{Abstract}

Introduction and objectives: omega-3 acids are polyunsaturated fatty acids that have to be provided in a preformed way to diet, being in oily fish which are in most. Omega-3 fatty acids are found in neuronal lipid membranes to a great extent, providing stability, and optimizing synaptic communication. A diet low in omega-3 acids, and this is a trend in Western eating habits in recent times, could be a risk factor for the development of cognitive disorders. This review attempts to assess the influence of omega-3 fatty acids in dementia (also referring to cognitive decline and mild cognitive impairment situation).

Development: neurobiological, epidemiological and clinical data of most important publications in this regard are presented.

Conclusions: a majority of epidemiological studies and some clinical trials found that a diet rich in omega-3 acids may decrease the incidence of dementia, could be correlated with a slowing of normal cognitive decline associated with age, or reduce the cognitive impairment in elderly patients diagnosed with mild cognitive impairment. However, currently available data do not support their utility to in established dementia. Frequent consumption of fatty fish is highly recommended, considering their intake as a supplement in the event that this does not happen.

(Alzheimer. Real Invest Demenc. 2011;49:12-18)

Keywords: omega-3 acids, dementia, depression, Alzheimer's disease, polyunsaturated fats, psychiatry in old age. 


\section{Introducción}

Los ácidos omega-3 son grasas poliinsaturadas de cadena larga cuya fuente se origina en el entorno marino o el reino vegetal. Estas grasas no las sintetiza el cuerpo humano, por lo que deben incorporarse a través la dieta. Determinados aceites de vegetales, como la nuez, la semilla de soja, el lino o la canola, contienen diferentes cantidades de ácido alfa linoleico (ALA), que el hígado transforma, en un pequeño porcentaje (5-15\%), en cadenas más largas, que conforman los ácidos omega-3: ácido eicosapentanoico (EPA) y ácido docosahexaenoico (DHA). De esta manera, la vía principal de aporte de estas grasas es a través de una dieta que posea estos ácidos de cadena larga poliinstaturada de manera preformada, que se hallan predominantemente en lo que denominamos las variedades de pescado azul.

Los ácidos omega-3 parecen ejercer una acción muy importante en las membranas neuronales, especialmente en las regiones sinápticas de las mismas (y en mayor grado, en áreas de sustancia gris), donde se acumulan en mayor proporción (y llegan a representar el 15\% de la composición total de ácidos grasos en el cerebro). Estas grasas son un componente esencial de la membrana fosfolipídica, por lo que su importancia es vital para la estabilidad de la estructura dinámica y la actividad funcional de las membranas neuronales ${ }^{1}$, pues pueden alterar la fluidez de la membrana lipídica (desplazando al colesterol de la misma) y promover la plasticidad sináptica, que es esencial para los procesos de aprendizaje, memoria y otros procesos cognitivos $^{2}$. Asimismo, actúan como fuentes de comunicación para segundos mensajeros entre neuronas ${ }^{1,3}$, mejoran el acoplamiento de las proteínas $\mathrm{G}$ que intervienen en muchas vías de transducción de señales ${ }^{4}$ e intervienen en funciones de transcripción directa relacionada con los lípidos 5 .

Recibido para su publicación: 2 de diciembre de 2010. Aceptado para su publicación: 2 de febrero de 2011. Correspondencia: J. Caballer García.

E-mail: caballergarcia@hotmail.com
Pese a la recomendación de que la relación entre la ingesta de ácidos omega-6 y omega-3 sea de aproximadamente 4:1, como venía sucediendo hasta principios del siglo $\mathrm{XX}^{6}$, las dietas ricas en ácidos grasos insaturados (omega-6), debido al alto consumo de aceites de origen vegetal por parte de la sociedad occidental, hace que esta relación haya llegado a ser de 20:17. Ya que uno de cada tres ácidos grasos en el sistema nervioso central son de cadena larga poliinsaturada, podría llegarse a pensar que un inadecuado balance entre éstos conllevaría alteraciones de tipo neuropsiquiátrico a nivel central ${ }^{8}$. Un consumo alto de ácidos omega-3 se ha asociado con incrementos de volumen de materia gris del circuito corticolímbico, por lo que sus efectos se objetivarían a nivel estructural en regiones íntimamente relacionadas con procesos cognitivos ${ }^{2}$. El propósito de este artículo de revisión es valorar el papel que tienen los ácidos omega-3 en la función cognitiva, ya sea en pacientes con demencia establecida, o en estadios más iniciales o de deterioro cognitivo ligero, así como en el declive cognitivo no patológico.

\section{Declive/deterioro cognitivo y ácidos omega-3}

Con el envejecimiento, especialmente en ancianos con la enfermedad de Alzheimer, parece que los niveles de DHA en el cerebro tienden a disminuir ${ }^{9},{ }^{10}$; este dato sugiere que este descenso contribuye tanto al deterioro de la memoria como a la afectación de otras funciones cognitivas.

Los ácidos grasos de cadena larga omega-3 poseen múltiples mecanismos de acción en el cerebro y el sistema vascular que podrían generar cierta protección contra el declive cognitivo y la demencia.

Como factores de protección cardiovascular, reducen el riesgo de aparición de enfermedades coronarias $^{11}$. En un metaanálisis reciente se ha observado que las grasas de pescado pueden conllevar una reducción de la muerte por enfermedad coronaria hasta en un $36 \%{ }^{12}$. También actúan como factores de protección cerebrovascular: se ha correlacionado su consumo con una menor incidencia significativa de lesiones 
en la sustancia blanca profunda ${ }^{13}$ y una disminución del riesgo de accidentes cerebrovasculares, particularmente los de tipo isquémico ${ }^{14}$.

Es sabido que existen procesos inflamatorios implicados en el desarrollo de la demencia. Los ácidos grasos omega-3 podrían figurar como agentes protectores por su acción antiinflamatoria, reduciendo la producción de ácido araquidónico y sus metabolitos, del factor de necrosis tumoral o de ciertos tipos de interleuquinas $^{15,16}$.

El DHA participaría en mecanismos de neuroprotección, como factor antioxidante ${ }^{17} \mathrm{o}$ antiapoptótico a través de la neuroprotectina NPD1 (metabolito del DHA por acción de la lipooxigenasa) $)^{5}$, o en mecanismos de neurogénesis, favoreciendo la producción de factores neurotrópicos como el factor neurotrófico de origen cerebral (BDNF) ${ }^{18}$.

Asimismo, los ácidos omega-3 podrían limitar de forma directa ciertos procesos que se relacionan con la neuropatología de la enfermedad de Alzheimer. Diversos trabajos $^{19-25}$ han hallado que el DHA podría influir favoreciendo una menor producción y acumulación del péptido tóxico A beta amiloide, por diferentes mecanismos de acción. El DHA también podría ejercer cierta función neuroprotectora, protegiendo a las sinapsis de la toxicidad de los elementos de la proteína A beta amiloide ${ }^{26-28}$. Junto con el uso de antioxidantes/vitaminas, estas acciones podrían verse potenciadas.

Desde el punto de vista epidemiológico/clínico se presentan una serie de estudios que nos podrían ofrecer información relevante a la hora de examinar la posible influencia de estas grasas en el funcionamiento cognitivo de ancianos no demenciados, prevenir o retrasar el inicio de un cuadro demencial, o favorecer el rendimiento cognitivo de los ancianos con diagnóstico de demencia, por lo que se intentará aproximar una respuesta a las siguientes cuestiones.

\section{¿Influye la toma de ácidos omega-3 en el funcionamiento cognitivo de ancianos no demenciados?}

En este sentido, contamos con el estudio prospectivo de cohortes $(\mathrm{N}=3.718)$ de Morris y cols. ${ }^{29}$, de 6 años de seguimiento, en el que la toma de pescado al menos una vez a la semana disminuía la tasa de declive cognitivo (medido el cambio en el declive mediante un modelo mixto) en un 10-13\% anual, ajustados estos datos al análisis multivariante (sexo, edad, raza, nivel educativo, actividad cognitiva, actividad física, consumo de alcohol y la cantidad total energética ingresada mediante la dieta).

Van Gelder y cols. ${ }^{30}$ publicaron otro estudio prospectivo de cohortes ( $\mathrm{N}=210$, varones), en el que se objetivó de forma estadísticamente significativa un menor avance del declive cognitivo (medido por el Minimental test de Folstein), al cabo de 5 años, de 0,3 puntos de media en los ancianos que consumían pescado (equivalente a la toma de al menos $380 \mathrm{mg}$ de $\mathrm{EPA}+\mathrm{DHA}$ ) frente a la pérdida de 1,2 puntos de media en los no consumidores, datos que se apoyaban en el análisis multivariante (edad, nivel educativo, consumo de alcohol y de tabaco, actividad física, ingesta calórica y funcionamiento cognitivo basal).

Heude y cols. ${ }^{31}$ presentaron un estudio prospectivo de cohortes $(\mathrm{N}=246)$ de 4 años de seguimiento. Poseer una elevada proporción de ácidos omega-3 (con respecto a otras grasas) en sangre se asoció de forma estadísticamente significativa con un menor declive cognitivo (OR=1,91; $\mathrm{CI}=1,16-3,15)$.

Otro estudio prospectivo de cohortes $(\mathrm{N}=2251)^{32}$ de Beydoun y cols., de 8 años de seguimiento, encontró que los niveles plasmáticos elevados de ácidos omega-3 se asocian con un menor riesgo de deterioro en la fluencia verbal.

En sentido negativo, disponemos de un reciente ensayo clínico randomizado y doble ciego $(\mathrm{N}=302)$ realizado por Van de Rest y cols. ${ }^{33}$, de 26 semanas de duración, en el que los suplementos de ácidos omega-3 no suponían una ventaja frente al declive cognitivo con respecto a la toma de placebo.

\section{¿Influye la toma de ácidos omega-3 en la incidencia de demencia?}

Kalmijn y cols. ${ }^{34}$ publicaron un estudio prospectivo de cohortes ( $\mathrm{N}=5.386)$, de 2 años de duración, en el que el consumo de pescado, concretamente en cantidades tota- 
les superiores a los $18,5 \mathrm{~g}$ por día, se asociaba de forma muy significativa $(\mathrm{P}=0,005)$ a una menor tasa de incidencia de la enfermedad de Alzheimer $(\mathrm{RR}=0,3)$, ajustados estos datos en el análisis multivariante para la edad, el sexo, la educación y la cantidad energética total de la dieta. Este resultado fue negativo en el análisis de pacientes con demencia asociada a un componente vascular.

El trabajo de Barberger-Gateau y cols. ${ }^{35}$ se trata de otro estudio prospectivo de cohortes $(\mathrm{N}=1.416)$ de 7 años de seguimiento, en el que la toma de pescado azul al menos una vez a la semana reducía la incidencia de casos de demencia de forma significativa en el análisis ajustado a la edad, aunque se perdía esta significación estadística en el análisis multivariante. Los mismos autores ${ }^{36}$, en otro estudio prospectivo de cohortes $(\mathrm{N}=8.085)$ de 4 años se seguimiento, hallaron que el riesgo reducido de padecer demencia en cualquiera de sus variantes (cuando el consumo de pescado era al menos semanal) sólo aparecía en los no portadores del alelo ApoE \&4. Asimismo, el consumo importante de ácidos omega-6, o no compensado por un consumo elevado de ácidos omega-3, actuaba como un factor de riesgo para padecer demencia.

La protección que el consumo de pescado azul, al menos dos veces por semana, ofrece frente a la aparición de la enfermedad de Alzheimer también es válida únicamente para los no portadores del alelo ApoE $\varepsilon 4$, según el estudio prospectivo de cohortes $(\mathrm{N}=2.233)$ de Huang y cols. ${ }^{37}$.

Morris y cols. ${ }^{38}$ publicaron un estudio prospectivo de cohortes $(\mathrm{N}=815)$ de 4 años de duración, en que el consumo de grasas de pescado, concretamente en cantidades totales superiores a los $1,75 \mathrm{~g}$ por día, se asociaba de forma significativa a una menor tasa de incidencia de la enfermedad de Alzheimer $(\mathrm{RR}=0,4)$, datos que se apoyaban en el análisis multivariante (edad, sexo, raza, educación, toma de vitamina E y de otras grasas, enfermedad cardiovascular y genotipo ApoE ع4). Sin embargo, en un análisis pormenorizado para valorar la influencia de cada tipo de ácido graso por separado, la única sustancia añadida exógenamente a la dieta que, de forma estadísticamente significativa, aparecía como factor protector frente a la aparición de enfermedad de Alzheimer fue el DHA (en cantidades supe- riores a los 0,07 g por día), y no fueron significativas las aportaciones exógenas de ALA y EPA.

Teniendo en cuenta esta vez los niveles plasmáticos de ácidos grasos, en concreto los niveles de DHA, Schaefer y cols. ${ }^{39}$ hallaron en otro estudio prospectivo de cohortes ( $\mathrm{N}=899)$, de 9,1 años de seguimiento, que los individuos que pertenecían al cuartil más alto de la muestra con respecto al contenido de DHA porcentual sobre el total de ácidos grasos en sangre (mayor del 4,5\%, correspondiéndose estos niveles con una toma diaria de DHA mayor de 0,18 g/día o una media de consumo de pescado de tres veces por semana), presentaban un menor riesgo de aparición de demencia de cualquier causa o de enfermedad de Alzheimer que los pertenecientes a los tres cuartiles inferiores, ajustados estos datos al sexo, la edad, la educación, los niveles plasmáticos de homocisteína y el genotipo ApoE $\varepsilon 4$. Al ajustarse sobre el genotipo el nivel de significación aumentaba en el grupo de no portadores del alelo e4 (siendo este grupo un $78 \%$ de la muestra).

Del mismo modo, Samieri y cols. ${ }^{40}$ valoraron en este estudio prospectivo de cohortes ( $\mathrm{N}=1.214)$, de 4 años de duración, los niveles de ácidos grasos en sangre. Los niveles de EPA plasmáticos se asociaron de forma inversa con la incidencia de demencia e independientemente de la presencia de clínica depresiva, ajustados estos datos en el análisis multivariante para el sexo, la edad, el nivel educativo, la presencia de diabetes, el genotipo ApoE ع4, y los niveles plasmáticos de vitamina E y triacilglicerol. Además, una mayor proporción de ácidos omega-6 sobre ácidos omega-3 o de acido araquidónico sobre DHA en sangre se asoció a una mayor incidencia de demencia. El estudio de la relación entre el DHA y la cantidad total de ácidos omega-3 con respecto a la incidencia de demencia no arrojó datos significativos en el modelo multivariante.

\section{¿Produce algún efecto beneficioso la toma de ácidos omega-3 en pacientes con diagnóstico de enfermedad de Alzheimer?}

Terano y cols. ${ }^{41}$ publicaron un pequeño ensayo clínico en el cual 20 pacientes diagnosticados de demen- 
cia vascular fueron asignados de forma randomizada a un grupo que recibía un suplemento de DHA (430 mg/día) y otro grupo control con placebo, durante 12 meses. Los pacientes que recibieron DHA experimentaron una reducción en la tasa de declive cognitivo (medida por el Minimental test de Folstein) a lo largo de los 3 y 6 primeros meses de seguimiento, aunque no obtuvieron este resultado significativo a los 6 meses.

Kotani y cols. ${ }^{42}$ realizaron un ensayo clínico doble ciego de 90 días de seguimiento, contando con un grupo de 39 pacientes, de los cuales 21 había sido diagnosticados de deterioro cognitivo ligero, 8 de enfermedad de Alzheimer y 10 con lesiones cerebrales (accidente cerebrovascular en los últimos 5 años). Un grupo recibió $240 \mathrm{mg}$ de DHA, mientras que el grupo control recibió $240 \mathrm{mg}$ de aceite de oliva. Al inicio y final de este período se administraron diversas baterías neuropisocológicas y no se halló ninguna mejoría significativa en los pacientes con enfermedad de Alzheimer. En los pacientes diagnosticados de deterioro cognitivo ligero mejoraron de forma significativa las puntuaciones en las escalas de atención y memoria inmediata, aunque no así en la memoria remota. Los pacientes con diagnostico de accidente cerebrovascular mejoraron los resultados en ambos tipos de memoria.

Disponemos de otro ensayo clínico randomizado doble ciego de mayor tamaño (N=174) de Freund-Levi y cols. ${ }^{43}$ sobre pacientes diagnosticados de enfermedad de Alzheimer, en el cual no se objetivaron diferencias significativas en la tasa de deterioro cognitivo (medida por el Minimental test de Folstein) entre el grupo de pacientes que recibió placebo y el que recibió un suplemento diario de DHA (430 mg) y EPA $(150 \mathrm{mg})$ a los 6 meses. Sin embargo, al ser estudiado el subgrupo de pacientes con diagnóstico de enfermedad de Alzheimer muy leve (puntuación mayor de 27 en el Minimental, pudiéndose considerar a estos pacientes realmente como diagnosticados de deterioro cognitivo ligero) se objetivó en los que recibían el suplemento una significativa mejoría de 2,1 puntos en el test, frente a ninguna mejoría en el grupo control.

Chiu y cols. ${ }^{44}$ publicaron un ensayo clínico randomizado doble ciego $(\mathrm{N}=35)$ de 24 semanas de dura- ción. Los pacientes con diagnóstico de deterioro cognitivo ligero que recibieron un suplemento diario de $1.080 \mathrm{mg}$ de EPA y de $720 \mathrm{mg}$ de DHA mejoraron la puntuación en test de valoración cognitiva administrado (ADAS-cog) en 3,23 puntos de media, mientras que el grupo control empeoró el resultado en 0,37 puntos, siendo este resultado estadísticamente significativo y ajustado para el sexo, la edad y el nivel educativo. No se objetivaron diferencias para los pacientes diagnosticados de enfermedad de Alzheimer con respecto al grupo control en el rendimiento cognitivo al final del estudio.

Finalmente, contamos con los resultados de un reciente estudio, de Nelson y cols. ${ }^{45}$, tratándose de un ensayo clínico doble ciego ( $\mathrm{N}=485)$, de 24 semanas de duración, sobre pacientes diagnosticados de deterioro cognitivo ligero. Los pacientes que recibieron un suplemento de 900 mg de DHA al día experimentaron una significativa mejoría en la memoria episódica y el aprendizaje (medidas por el test de aprendizaje emparejado PAL, de la batería neuropsicológica automatizada Cambridge CANTAB), hallándose una mejoría neta de 3,4 años en estos procesos cognitivos con respecto a los pacientes control que recibieron placebo.

\section{Conclusiones}

La importancia de las funciones a nivel central de los ácidos omega-3 parece tener sustento desde el plano neurobiológico, especialmente en sus acciones como estabilizador de las membranas neuronales y, en consecuencia, optimizando diversos sistemas de comunicación. Asimismo, los ácidos omega-3 parecen tener una acción neuroprotectora (por mecanismos antiinflamatorios, antioxidativos, antiapoptóticos y cardioprotectores), participando también en la neurogénesis e incluso interviniendo positivamente en ciertos procesos neurodegenerativos.

El consumo de grasas de pescado también se ha correlacionado con un enlentecimiento del declive cognitivo en ancianos sanos, e incluso en aquellos diagnosticados de deterioro cognitivo ligero, y puede además influir disminuyendo la incidencia del diagnóstico 
de demencia. Sin embargo, los datos existentes en la actualidad requieren más estudios que permitan encontrar una utilidad de los mismos en demencias ya establecidas.

En definitiva, parece que el consumo de pescado, especialmente de la variedad azul, es muy recomendable al menos entre dos y tres veces por semana. $\mathrm{Si}$ por cualquier razón esto no fuera así, debería considerarse su aportación a modo de suplemento.

\section{Bibliografía}

1. McNamara RK, Ostrander M, Abplanalp W, Richtand NM, Benoit S, Clegg D. Modulation of phosphoinositideprotein kinase $\mathrm{C}$ signal transduction by omega-3 fatty acids: Implications for the pathophysiology and treatment of recurrent neuropsychiatric illness. Prostaglandins Leukot Essent Fatty Acids. 2006;74(4-5):237-57.

2. Jicha GA, Markesbery WR. Omega-3 fatty acids: potential role in the management of early Alzheimer's disease. Clin Interv Aging. 2010;7(5):45-61.

3. Yehuda S, Rabinovitz S, Mostofsky DI. Essential fatty acids and the brain: from infancy to aging. Neurobiol Aging. 2005;26 Suppl 1:98-102.

4. Arvindakshan M, Sitasawad S, Debsikdar V, Ghate M, Evans D, Horrobin DF, et al. Essential polyunsaturated fatty acid and lipid peroxide levels in never-medicated and medicated schizophrenia patients. Biol Psychiatry. 2003; 53(1):56-64.

5. Uauy R, Dangour AD. Nutrition in brain development and aging: role of essential fatty acids. Nutr Rev. 2006;64:S24S33.

6. Cordain L, Eaton SB, Sebastian A, Mann N, Lindeberg S, Watkins BA, et al. Origins and evolution of the Western diet. Health implications for the 21 st century. Am J Clin Nutr. 2005;81:341-54.

7. Simopouloaus AP. Importance of the ratio of omega-6/ omega-3 essential fatty acids: evolutionary aspects. Worl Rev Nutr Diet 2003;82:1-22.

8. Logan AC. Omega-3 fatty acids and major depression: a primer for mental health professional. Lipids Health Dis. 2004;3:25.

9. Conquer JA, Tierney MC, Zecevic J, Bettger WJ, Fisher RH. Fatty acid analysis of blood plasma of patients with Alzheimer's disease, other types of dementia, and cognitive impairment. Lipids. 2000;35:1305-12.

10. Tully AM, Roche HM, Doyle R, Fallon C, Bruce I, Lawlor B. Low serum cholesteryl ester-docosahexaenoic acid levels in Alzheimer's disease: a case-control study. Br J Nutr. 2003;89:483-9.
11. Wang C. n-3 fatty acids from fish or fish-oil supplements, but nor alpha linolenic acid, benefit cardiovascular disease outcomes in primary and secondary prevention studies: a systematic review. Am J Clin Nutr. 2006;84:5-17.

12. Mozaffarian D, Rimm EB. Fish intake, contaminants, and human health: evaluating the risks and the benefits. JAMA. 2006;296:1885-99.

13. Virtanen JK, Siscovick DS, Longstreth WT Jr, Kuller LH, Mozaffarian D. Fish consumption and risk of subclinical brain abnormalities on MRI in older adults. Neurology. 2008;71(6):439-46.

14. He K, Song Y, Daviglus ML, Liu K, Van Horn L, Dyer AR, et al. Fish consumption and incidence of stroke: a metaanalysis of cohort studies. Stroke. 2004;35(7):1538-42.

15. Vedin I, Cederholm T, Freund Levi Y, Basun H, Garlind A, Faxén Irving $\mathrm{G}$, et al. Effects of docosahexaenoic acid-rich n-3 fatty acid supplementation on cytokine release from blood mononuclear leukocytes: the OmegAD study. Am J Clin Nutr. 2008;87(6):1616-22.

16. Tassoni D, Kaur G, Weisinger RS, Sinclair AJ. The role of eicosanoids in the brain. Asia Pac J Clin Nutr. 2008; 17(Suppl.):220-8.

17. Yavin E, Brand A, Green P. Docosahexaenoic acid abundance in the brain: a biodevice to combat oxidative stress. Nutr Neurosci. 2002;5:149-57.

18. Rao JS, Ertley RN, Lee HJ, DeMar JC Jr, Arnold JT, Rapoport SI, et al. Omega-3 polyunsaturated fatty acid deprivation in rats decreases frontal cortex BDNF via a p38 MAPK-dependent mechanism. Mol Psychiatry. 2007;12(1):36-46.

19. Ma QL, Teter B, Ubeda OJ, Morihara T, Dhoot D, Nyby $\mathrm{MD}$, et al. Omega-3 fatty acid docosahexaenoic acid increases SorLA/LR11, a sorting protein with reduced expression in sporadic Alzheimer's disease (AD): relevance to AD prevention. J Neurosci. 2007;27(52):14299307.

20. Cole GM, Ma QL, Frautschy SA. Omega-3 fatty acids and dementia. Prostaglandins Leukot Essent Fatty Acids 2009; 81(2-3):213-21.

21. Ma QL, Yang F, Rosario ER, Ubeda OJ, Beech W, Gant DJ, et al. Beta-amyloid oligomers induce phosphorylation of tau and inactivation of insulin receptor substrate via cJun N-terminal kinase signaling: suppression by omega-3 fatty acids and curcumin. J Neurosci. 2009 Jul 15;29(28): 9078-89.

22. Sahlin C, Pettersson FE, Nilsson LN, Lannfelt L, Johansson AS. Docosahexaenoic acid stimulates non-amyloidogenic APP processing resulting in reduced Abeta levels in cellular models of Alzheimer's disease. Eur J Neurosci. 2007;26(4):882-9.

23. Oksman M, Iivonen H, Hogyes E, Amtul Z, Penke B, Leenders I, et al. Impact of different saturated fatty acid, polyunsaturated fatty acid and cholesterol containing 
diets on beta-amyloid accumulation in APP/PS1 transgenic mice. Neurobiol Dis. 2006;23(3):563-72.

24. Lim GP, Calon F, Morihara T, Yang F, Teter B, Ubeda O, et al. A diet enriched with the omega-3 fatty acid docosahexaenoic acid reduces amyloid burden in an aged Alzheimer mouse model. J Neurosci. 2005;25(12):303240.

25. Green KN, Martinez-Coria H, Khashwji H, Hall EB, Yurko-Mauro KA, Ellis L, et al. Dietary docosahexaenoic acid and docosapentaenoicacid ameliorate amyloid-beta and tau pathology via a mechanism involving presenilin 1 levels. J Neurosci. 2007;27(16):4385-95.

26. Florent S, Malaplate-Armand C, Youssef I, Kriem B, Koziel V, Escanyé MC, et al. Docosahexaenoic acid prevents neuronal apoptosis induced by soluble amyloid-beta oligomers. J Neurochem. 2006;96(2):385-95.

27. Ma QL, Yang F, Calon F, Ubeda OJ, Hansen JE, Weisbart $\mathrm{RH}$, et al. p2l-activated kinase-aberrant activation and translocation in Alzheimer disease pathogenesis. J Biol Chem. 2008;283(20): 14132-43.

28. Lukiw WJ, Cui JG, Marcheselli VL, Bodker M, Botkjaer A, Gotlinger K, et al. A role for docosahexaenoic acid-derived neuroprotectin Dl in neural cell survival and Alzheimer disease. J Clin Invest. 2005;115(10):2774-83.

29. Morris MC, Evans DA, Tangney CC, Bienias JL, Wilson RS. Fish consumption and cognitive decline with age in a large community study. Arch Neurol. 2005;62(12): 1849-53.

30. Van Gelder BM, Tijhuis M, Kalmijn S, Kromhout D. Fish consumption, n-3 fatty acids, and subsequent 5-y cognitive decline in elderly men: the Zutphen Elderly Study. Am J Clin Nutr. 2007;85(4):1142-7.

31. Heude B, Ducimetière P, Berr C. Cognitive decline and fatty acids composition of erythrocyte membranes. Am J Clin Nutr. 2003;77(4):803-8.

32. Beydoun MA, Kaufman JS, Satia JA, Rosamond W, Folsom AR. Plasma n-3 fatty acids and the risk of cognitive decline in older adults: the Atherosclerosis Risk in Communities Study. Am J Clin Nutr. 2007;85(4): 1103-11.

33. Van de Rest O, Geleijnse JM, Kok FJ, Van Staveren WA, Hoefnagels WH, Beekman AT, et al. Effect of fish-oil supplementation on mental well-being in older subjects: a randomized, double-blind, placebo-controlled trial. Am J Clin Nutr. 2008;88(3):706-13.

34. Kalmijn S, Launer LJ, Ott A, Witteman JC, Hofman A, Breteler MM. Dietary fat intake and the risk of incident dementia in the Rotterdam study. Ann Neurol. 1997;42: 776-82.
35. Barberger-Gateau P, Letenneur L, Deschamps V, Peres K, Dartigues JF, Renaud S. Fish, meat, and risk of dementia: Cohort study. BMJ. 2002;325:932-3.

36. Barberger-Gateau P, Raffaitin C, Letenneur L, Berr C, Tzourio C, Dartigues JF, et al.Dietary patterns and risk of dementia: the Three-City cohort study. Neurology. 2007; 69(20):1921-30.

37. Huang TL, Zandi PP, Tucker KL, Fitzpatrick AL, Kuller LH, Fried LP, et al. Benefits of fatty fish on dementia risk are stronger for those without APOE epsilon4. Neurology. 2005;65(9):1409-14.

38. Morris MC, Evans DA, Bienias JL, Tangney CC, Bennet DA, Wilson RS, et al. Consumption of fish and n-3 fatty acids and risk of incident Alzheimer disease. Arch Neurol. 2003;60:940-6.

39. Schaefer EJ, Bongard V, Beiser AS, Lamon-Fava S, Robins $\mathrm{SJ}, \mathrm{Au} \mathrm{R}$, et al. Plasma phosphatidylcholine docosahexaenoic acid content and risk of dementia and Alzheimer disease: the Framingham Heart Study. Arch Neurol. 2006; 63(11):1545-50.

40. Samieri C, Féart C, Letenneur L, Dartigues JF, Pérès K, Auriacombe S, et al. Low plasma eicosapentaenoic acid and depressive symptomatology are independent predictors of dementia risk. Am J Clin Nutr. 2008;88(3):714-21.

41. Terano T, Fujishiro S, Ban T, Yamamoto K, Tanaka T, Noguchi Y, et al. Docosahexaenoic acid supplementation improves the moderately severe dementia from thrombotic cerebrovascular diseases. Lipids. 1999;34 Suppl: 345-6.

42. Kotani S, Sakaguchi E, Warashina S, Matsukawa N, Ishikura Y, Kiso Y, et al. Dietary supplementation of arachidonic and docosahexaenoic acids improves cognitive dysfunction. Neurosci Res. 2006;56(2):159-64.

43. Freund-Levi Y, Eriksdotter-Jönhagen M, Cederholm T, Basun H, Faxén-Irving G, Garlind A, et al. Omega-3 fatty acid treatment in 174 patients with mild to moderate Alzheimer disease: OmegAD study: a randomized doubleblind trial. Arch Neurol. 2006;63(10):1402-8.

44. Chiu CC, Su KP, Cheng TC, Liu HC, Chang CJ, Dewey $\mathrm{ME}$, et al. The effects of omega-3 fatty acids monotherapy in Alzheimer's disease and mild cognitive impairment: a preliminary randomized double-blind placebo-controlled study. Prog Neuropsychopharmacol Biol Psychiatry. 2008; 32(6): 1538-44

45. Yurko-Mauro K, McCarthy D, Rom D, Nelson EB, Ryan AS, Blackwell A, et al. Beneficial effects of docosahexaenoic acid on cognition in age-related cognitive decline. Alzheimers Dement. 2010 Nov;6(6):456-64. 